\section{PREVALENCE AND CLINICAL CORRELATES OF PATIENT-REPORTED AUTONOMIC SYMPTOMS AMONG SUBJECTS WITH COPD}

Jibril Mohammed ${ }^{1,2}$, Eric Derom ${ }^{3}$, Jessica Van Oosterwijck ${ }^{1,4}$, Hellen da Silva ${ }^{1}$, Patrick Calders ${ }^{1}$
Bayero University Kano

1 Dept. of Rehabilitation Sciences and Physical Therapy, Ghent University, Belgium; 2 Dept. Of Physiotherapy, Bayero University Kano, Nigeria; 3 Dept. of Respiratory Medicine Ghent University, Belgium; 4 Research Foundation Flanders (FWO) Brussels, Belgium.

\begin{abstract}
Alms
The objective of this study was to assess autonomic symptoms in subjects with COPD.

(I) Are autonomic symptoms prevalent in subjects with COPD?

(II) What are the most important clinical correlates of autonomic symptoms in subjects with COPD?
\end{abstract}

\section{METHODS}

COPD ( $n=53,39 \sigma^{\pi / 14}$ 우, Gold II-IV)

Age: $\quad 66 \pm 7$ years

Height: $\quad 1.68 \pm 8.2$ meters

Weight: $\quad 74.4 \pm 18.5$ kilograms

BMI: $\quad 26.1 \pm 6.3 \mathrm{Kg} / \mathrm{m}^{2}$

$\mathrm{FEV}_{1}$ (\%predicted): $1.17 \pm 0.50(41.9)$

FVC (\%predicted): $2.78 \pm 0.84(81.5)$

PEF (\%predicted): $3.65 \pm 1.3(50.2)$

ASP: autonomic symptom profile HADS and SF-36: general health status

Fatigue: checklist individual strength

Respiratory outcomes: FEV 1 , FVC, PEF \& dyspnea

Demographic variables: age, BMI, weight $\&$ height

\section{ANALYSIS}

Spearman rho coefficients (significance level: $\mathrm{P}<0,05$ )

Acknowledgements

Jibril Mohammed is on a PhD fellowship funded by the Tertiary Education Trust Fund, Nigeria. Jessica Van Oosterwijck is a postdoctoral research fellow funded by the Research Foundation Flanders (FWO) Brussels.

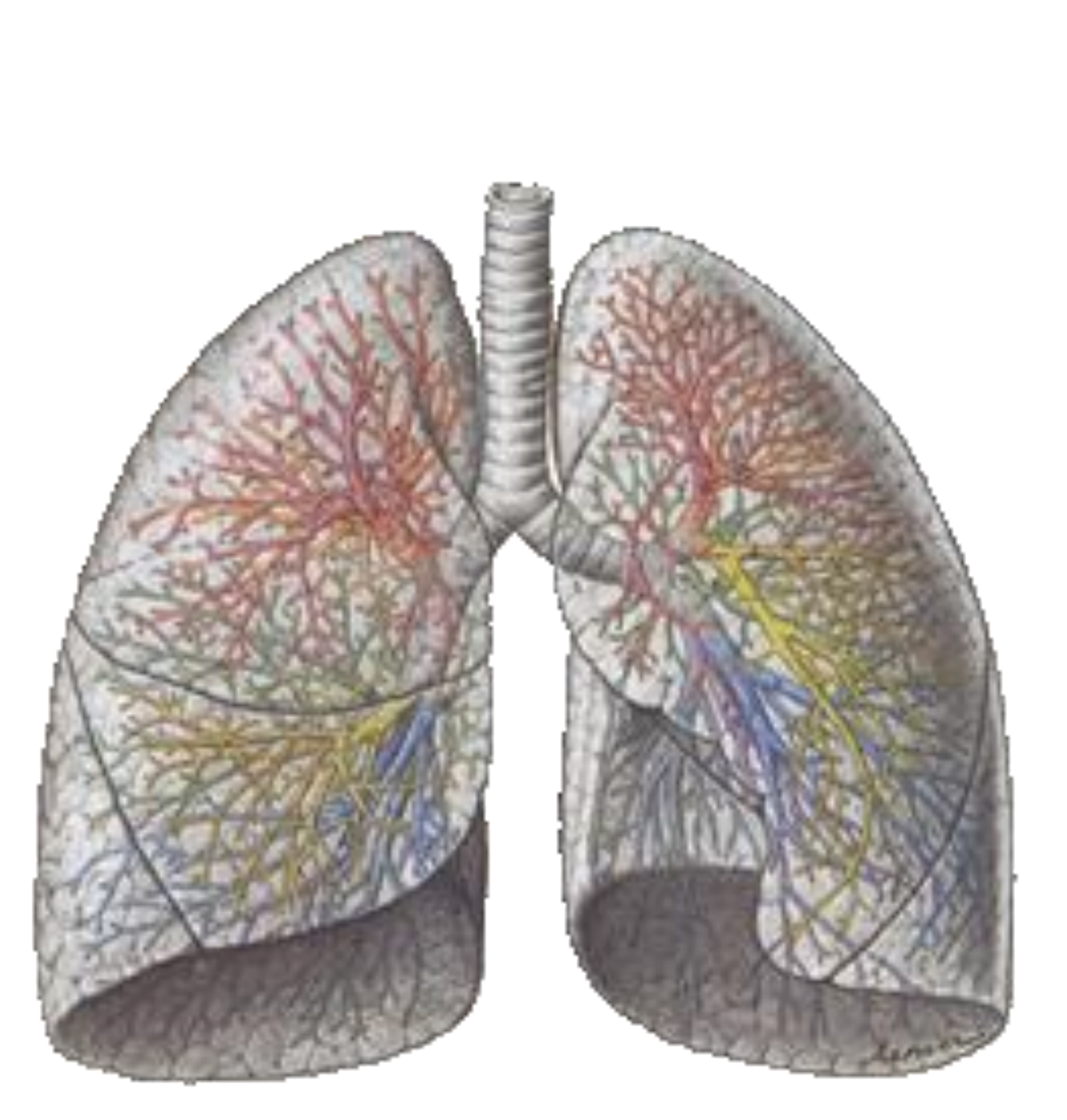

\begin{abstract}
RESULTS
Autonomic symptoms were present across all six domains of the ASP. These symptoms were mainly associated with high levels of anxiety, depression and worsened mental health $(p<0.05)$. The presence of severe autonomic symptoms was also associated with poor health status and higher levels of fatigue $(p<0.05)$. However, there were no correlations between the overall ASP scores and demographic or respiratory parameters, except for a diminished pupillomotor function that was mildly associated with reduced $\mathrm{FEV}_{1}$.
\end{abstract}

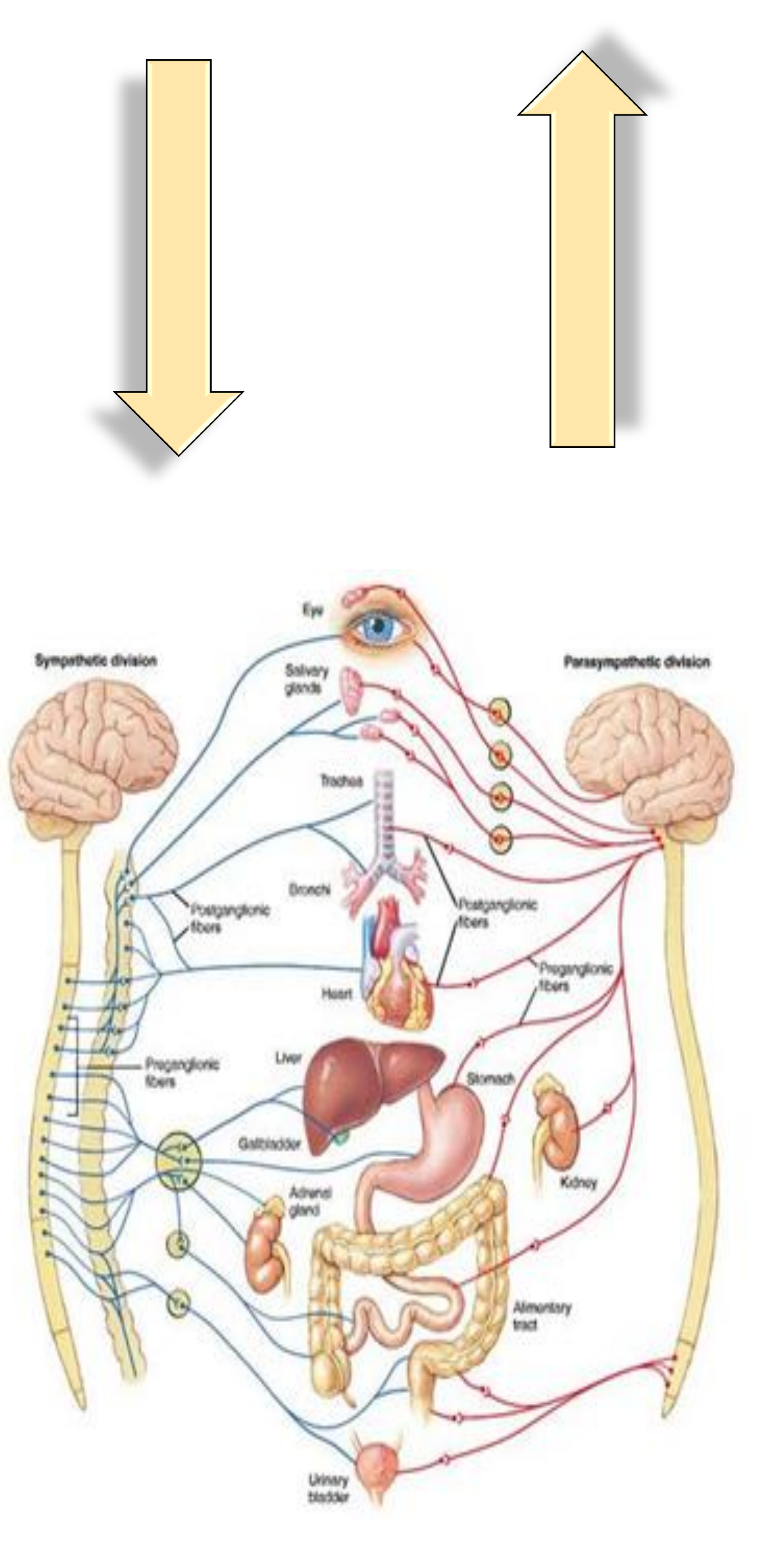

Contact details jibril.mohammed@ugent.be

\begin{tabular}{|c|c|c|c|c|c|c|c|}
\hline $\begin{array}{l}\text { Domains } \\
\text { /Clinical variables }\end{array}$ & $\begin{array}{l}\text { Orthostatic } \\
\text { intolerance }\end{array}$ & Vasomotor & Secremotor & $\begin{array}{l}\text { Gastro } \\
\text { intestinal }\end{array}$ & Bladder & $\begin{array}{l}\text { Papillo- } \\
\text { motor }\end{array}$ & $\begin{array}{l}\text { Total ASP } \\
\text { score }\end{array}$ \\
\hline \multicolumn{8}{|l|}{ Age } \\
\hline \multicolumn{8}{|l|}{ Height } \\
\hline \multicolumn{8}{|l|}{ Weight } \\
\hline \multicolumn{8}{|l|}{ BMI } \\
\hline $\mathrm{FEV}_{1}$ & & & & & & $0.286^{*}$ & \\
\hline \multicolumn{8}{|l|}{ FVC } \\
\hline \multicolumn{8}{|l|}{ PEF } \\
\hline Anxiety & & & $0.448^{*}$ & & $0.281^{*}$ & & $0.386^{*}$ \\
\hline Depression & & $0.288^{*}$ & $0.392^{*}$ & & & & $0.391^{*}$ \\
\hline Fatigue & & & $0.318^{*}$ & & $0.324 *$ & $0.312^{*}$ & $0.300^{*}$ \\
\hline PCS (SF-36) & & $-0.352^{*}$ & & & & & $-0.358^{*}$ \\
\hline $\operatorname{MCS}$ (SF-36) & $-0.291 *$ & & & $-0.309^{*}$ & & & $-0.385^{*}$ \\
\hline Dyspnea level & & & & & & & \\
\hline
\end{tabular}

\section{CONGLUSIONS}

Autonomic complaints are prevalent in subjects with COPD especially in those who have higher levels of anxiety, depression, fatigue, and poor general health. This highlights the need for screening autonomic function in COPD patients who fit this profile and their treatment should be adapted accordingly. 